\title{
Initial Tool Orientation Set-up for 5-Axis Flank Milling Based on Faceted Models
}

\author{
Gandjar Kiswanto ${ }^{1}$, Ario Sunar Baskoro ${ }^{1}$ and Eko Arif Syaefudin ${ }^{2}$ \\ ${ }^{1}$ Dept. of Mechanical Engineering, University of Indonesia, Depok, Indonesia \\ ${ }^{2}$ Dept. of Mechanical Engineering, Jakarta State University, East-Jakarta, Indonesia
}

\begin{abstract}
One of the factors affecting the effectiveness of machining time of 5-axis miling is the method being used. By using flank milling method, as one of the optimized processes to make a workpiece, the time required for the process becomes shorter.This research is aimed at developing the method for determining the initial orientation of the tool for a sculptured surface on the basis of faceted model. By determining cc-point as the basis for positioning the tool on the surface of the workpiece, the cutting direction is formed from the nearest cc-point in the XY flat plane direction of the faceted model at the spatial coordinate. The positioning of the tool is initially based on the Local Coordinate System developed by the cross product between the normal vector $n$ at each cc-point and cutting direction vector $F$ from one cc-point to the other. The cross product resulted is a tangent vector $T$ of the plane formed from the normal vector and cutting direction. The orientation of the tool is formed and defined by an inclination angle $(\alpha)$ and a screw angle $(\beta)$. Maximizing the cutting volume and avoiding gouging at each cc-point during the flank milling are carried out through optimal adjustment of these two rotational angles. Furthermore, when the adjustment of rotational angles cannot resolve the gouging, appropriate tool lifting along the normal vector is conductedhis method is very much applicable for flank milling having the basis of data in the form of faceted models.
\end{abstract}

\section{Introduction}

Based on the current development of technology, the manufacturing of a product having a complex surface such as aircraft components, automotive parts, molds and dies, etc. an effective and efficient method is required. In order to respond such challenge, the use of 5-axis milling machining becomes one of the solutions mainly selected by manufacturers [1-3].

For processing of planar surfaces in 5-axis milling machine, normally the flank milling or so-called peripheral milling method is used. Although the material removal rate (MRR) for the machining process using this method is very huge compared to the other methods, until now this method still has many disadvantages. One of the disadvantages is its being uncapable of processing a workpiece having a sculptured surface [4-6].

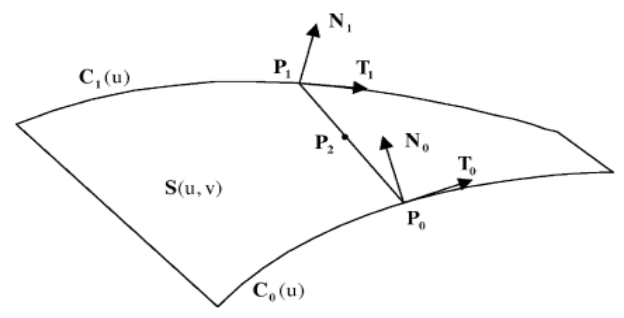

(a)

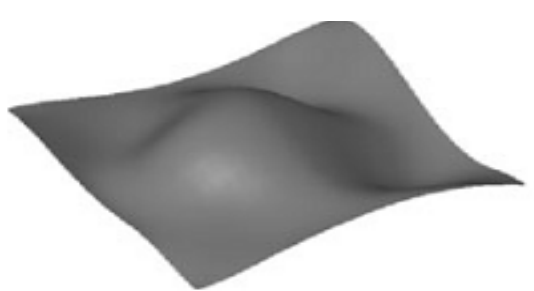

(b)

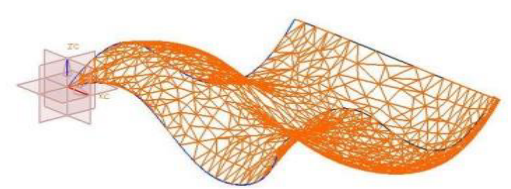

(c)

Figure 1. a. The development of tool track for flank milling [7], b. Sculptured surface, with many curve [7], c. STL of Sculptured Surface [8]

S Bedi and C Menzel [7] made a tool track for flank milling by controlling 2 constructing curves as shown in Figure 1. As for the other methods, that is SPO and DPO also control 2 reference pints to determine the position of the tool on the workpiece surface as described in Figure 2 below.

For a planar surface, parametric equation is commonly used for making the flank milling method by among others Chih-Hsing Chu and $\mathrm{Li}[3,6]$. 
In this study, the basis of data used is faceted model directly obtained from a CAD system with STL file format.The use of faceted model provides several advantages, which among others are easy to determine the normal vector at each triangle that willl later be used as the basis for calculating initial tool orientationat $\mathrm{CC}$ ccpoint for flank milling, where to calculation cc-point has be done on research before $[8,9]$. To determine the normal vector at eact cc-point, calculation is made using the following equation and is illustrated in Figure 2 below

$$
n=\frac{\sum_{l} S_{l} \boldsymbol{n}_{l}}{\sum_{l} S_{l}}
$$

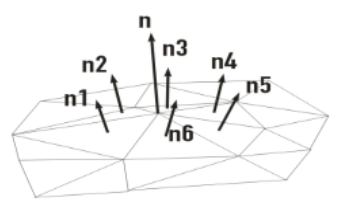

Figure 2. Calculating the cc-point normal vector (n), there is between the triangles

\section{Methodology}

In this study, determination of tool orientation for flank milling starts from calculation of the normal vector value at each cc-point. This can be described by the flow chart below.

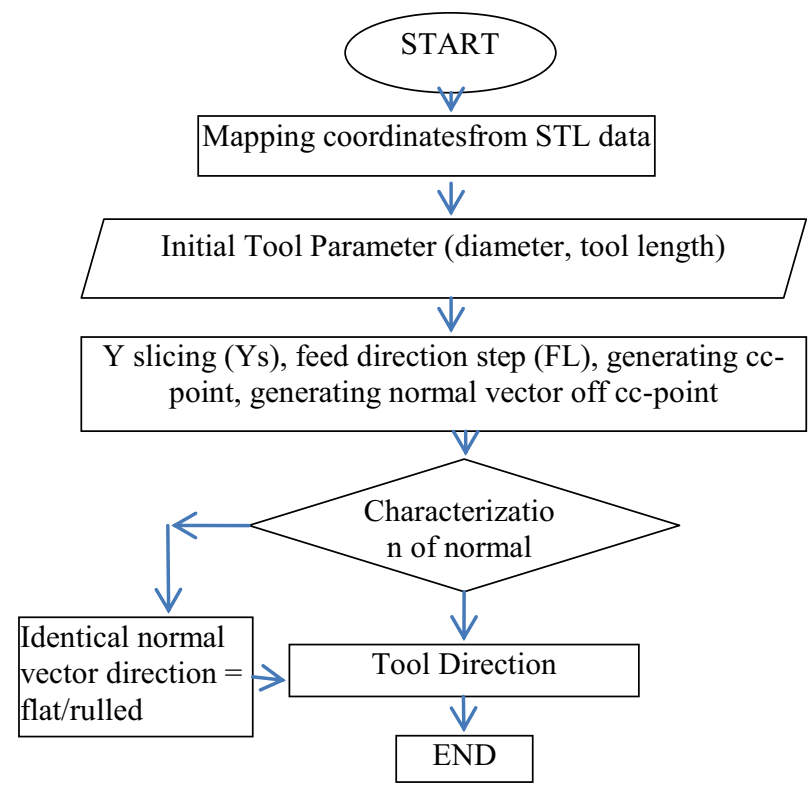

Figure 3. Flowchart of initial tool orientation set-up for flank milling.

To form a set of cc-points on a workpiece surface, the slicing plane method is applied in this study. The slice of the slicing plane (Ys) against each triable on the workpiece surface becomes the basis for the formation of this cc-point. Meanwhile, the position of cc-point at each triangle passed by the slicing plane will also be calculated based on the normal vector formed by each of the said triangles. This slicing planemethod will form am imaginary line, so that later the cutting direction in the machining process will follow this slicing plane. As described in Figure 4 below, the position of each ccpointon the faceted model is formed from the intersection between the slicing plane and the faceted model and thus result the cc-point coordinates $\mathrm{x}, \mathrm{y}$ and $\mathrm{z}$ using the following equation:

$$
\begin{gathered}
\frac{x-x 1}{x 2-x 1}=\frac{y-y 1}{y 2-y 1}=\frac{z-z 1}{z 2-z 1} \\
x=\frac{y-y 1}{y 2-y 1} x(x 2-x 1)+x 1 \\
z=\frac{y-y 1}{y 2-y 1} x(z 2-z 1)+z 1
\end{gathered}
$$

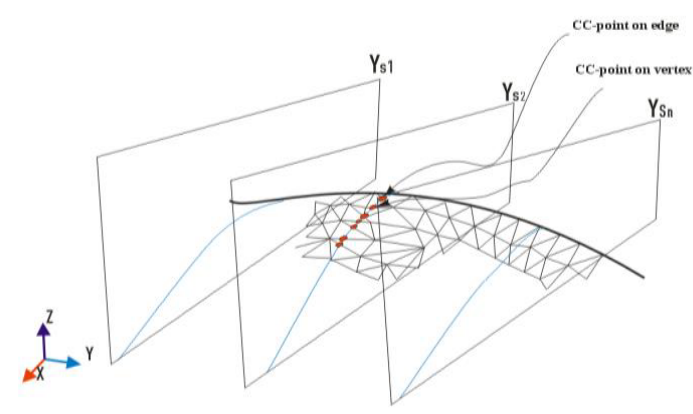

Figure 4. The development of cc-point using slicing plane method

This process can be computed by the psudocode below:

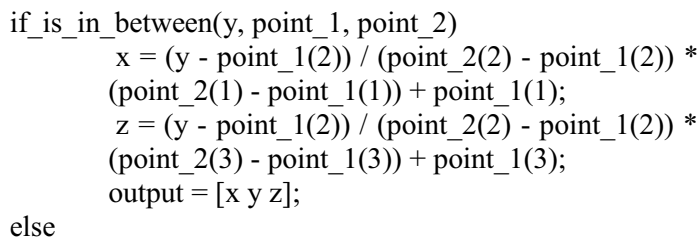

Meanwhile, to form a normal vector at each cc-point, based on the result of slicing plane method in the previous steps the psudocode used is as follows:

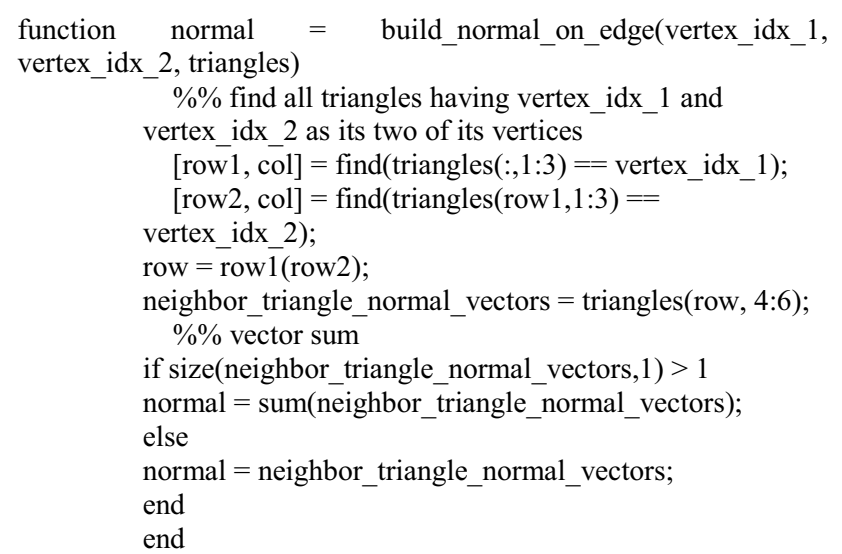

\section{The determination of initial tool orientation for flank milling}

Based on the previous explanation that the cc-point was obtained from the result of slicing at the triangles on the faceted model by the cutting plane (Ys) with the predetermined step-over (in Figure 4), the cc-point coordinates can be obtained. Then the normal vector at the cc-point can be calculated based on the average value of the vectors surrounding it and are indexed like what has been performed by Lauwers, Kiswanto, et al and illustrated in Figure 2. Each of the cc-points produces a 
normal vector and also feed direction vector as described in Figure 5, where the feed direction vector is a vector pointing from the current cc-point to the next ccpoint.Then, from each cc-point a Local Coordinate System can be formed with the axes being normal vectors $\underset{N}{\rightarrow}$, feed direction vector $\underset{F}{\rightarrow}$ and cross product between the normal vector and feed direction vector $\underset{T}{\rightarrow}=\underset{N}{\rightarrow} x \underset{F}{\vec{F}}$ ) [10, $11]$, as depicted in Figure 6.

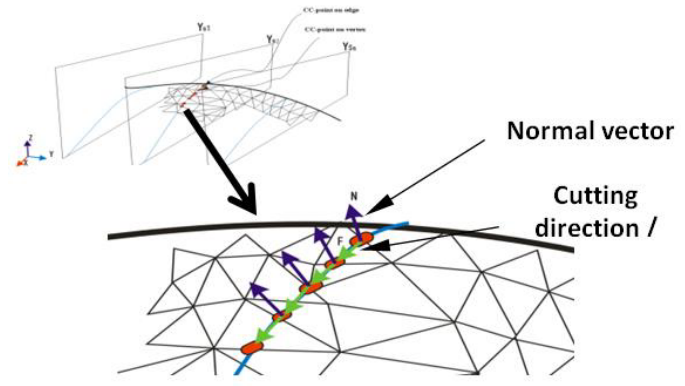

Figure 5. Determining normal vector $(N)$ and feed $n$ direction vector $(F)$

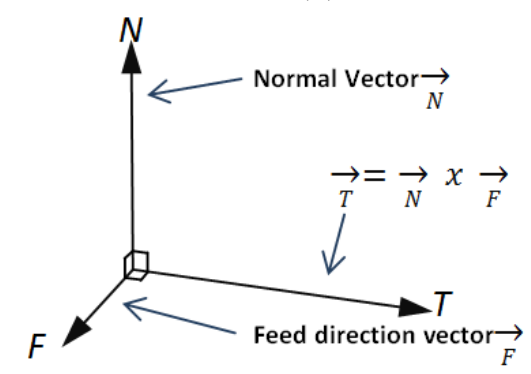

Figure 6. Local tool coordinate references

The tool orientation at this flank milling based on faceted model is formed by 2 angles, namely inclination angle $(\alpha)$ and screw angle $(\beta)$. Inclination angle $(\alpha)$ is an angle between tool axis vector and the normal vector $(\vec{N})$, whilethe screw angle $(\beta)$ is the angle tool axis vector and plane formed by Feed direction vector $(\overrightarrow{\boldsymbol{F}})$ and normal vector $(\overrightarrow{\boldsymbol{N}})$. Such angles ( $\alpha$ and $\beta$ ) are the tool orientation angles during the flank machining process. In order to avoid gouging, adjustments conducted to both angles, so that the gouging can be eliminated. Description about the rotation angles can be seen in Figure 7 . In the condition of initial position, the tool is placed in parallel with the Axis $\mathrm{T}$, in this case $\alpha$ has a value of $90^{\circ}$.

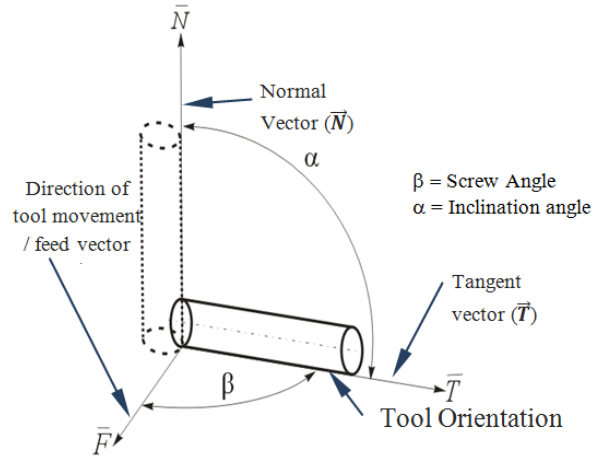

Figure 7. Tool orientation at the local coordinate system

Furthermore, in order to reduce the complexity in avoiding gouging during the flank machining operation, ( $\beta$ ) angle is adjusted to be always $90^{\circ}$, so that the tool orientation is mostly performed by the inclination angle $(\alpha)$ as illustrated in Figure 8.

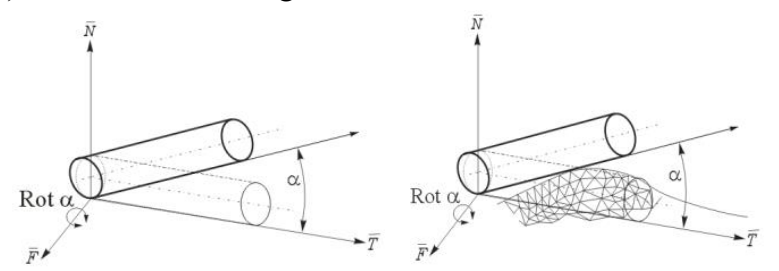

Figure 8. Tool inclination $(\alpha)$ to avoid gouging.

However, when the adjustment of tool orientation by inclination angle cannot resolve the gouging, an appropriate tool lifting along the normal vector must be conducted, as shown in Figure 9.

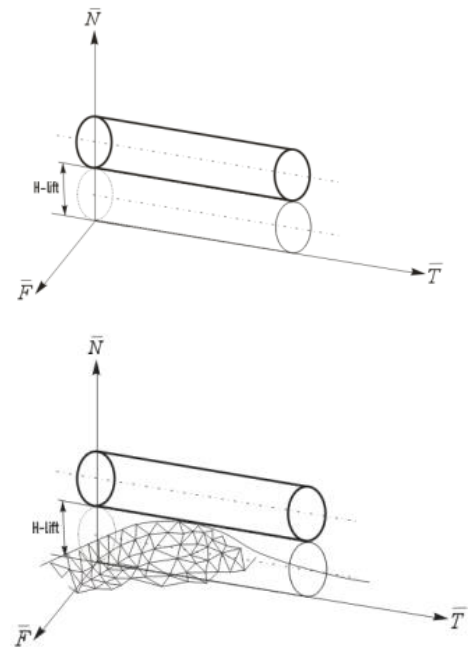

Figure 9. Tool Lifting to avoid gouging.

Figure 10 illustrates tool when avoiding gouging by inclining and lifting simultaneously.
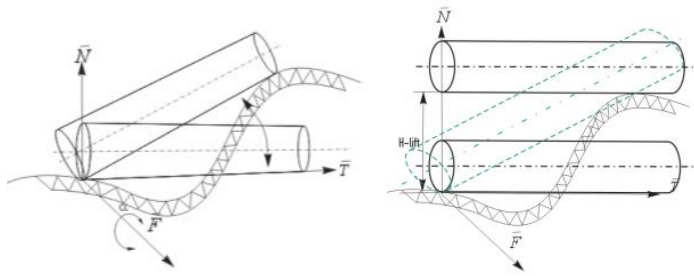

Figure 10. Tool conductinginclinationand lifting

Illustration of the result of the initialtool orientaion methode flank milling before gouging avoidance in this paper has been successfully and well applied in programming algorithm as described in Figure 11.

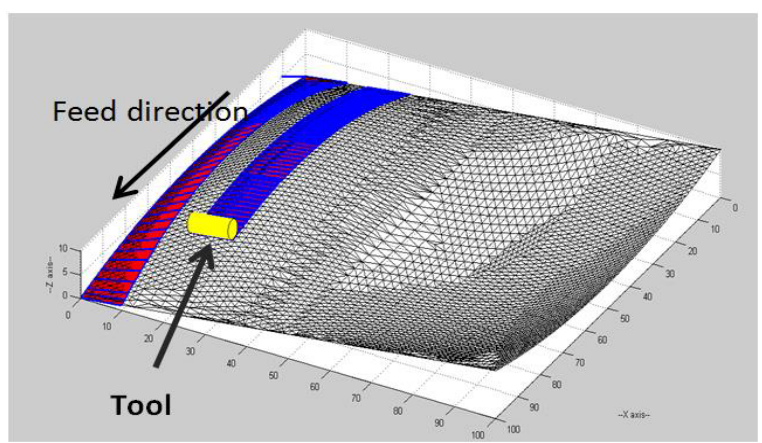

Figure 11. The tool position against the workpiece surface using flank mlling method. 


\section{Conclusions}

By the use of the faceted model as the basis for the method for determining the tool direction for this flank milling, the determination of the normal vector and the tool position can become very easy to use and very much applicable.The tool orientation direction is a result of cross product or tangential value of 2 vectors, that is normal vector and vector from the cutting direction. Meanwhile, the tool position against Cc-point is the outer side of the contact between the tool and workpiece surface. Thus, the center position of the tool is equal to the $\mathrm{Cc}$-point plus a half of the tool diamter against $\mathrm{Z}$ axis.

\section{References}

1. Li, L.L.B.C.F.L.C., Complexity analysis and calculation for sculptured surface in multi-axis CNC machining based on surface subdivision. Int $\mathrm{J}$ AdvManufTechnol, (2014). 71: 1433-1444 DOI 10.1007(s00170-013-5544-2).

2. Chaves-Jacob, J., G. Poulachon, and E. Duc, New approach to 5-axis flank milling of free-form surfaces: Computation of adapted tool shape.Computer-Aided Design, 41(12): p. 918-929 (2009)

3. Zhu, L., H. Ding, and Y. Xiong, Simultaneous optimization of tool path and shape for five-axis flank milling. Computer-Aided Design, 44(12): p. 12291234 (2012)

4. Bedi S, M.S., Menzel C. Flank milling with flat end milling cutters. (2003). Computer-Aided Design.
5. Kruth, G.K.B.L.J.-P., Gouging elimination through tool lifting in tool path generation for five-axis milling based on faceted models. Int J Adv Manuf Technol (2007). DOI 10.1007/s00170-005-0338-9: p. 16.

6. Chih-Hsing Chu, H.-T.H., Generation of reciprocating tool motion in 5-axis flank milling based on particle swarm optimization. International Journal of Machine Tools and Manufacture (C) Springer Science+Business Media, LLC 2010, 23: p. 1501 (2012)

7. Sanjeev Bedi, S.M., Cornelia Menzel, Flank milling with flat end milling cutters. Computer-Aided Design, Vol. 35, (2005): p. 293-300

8. Kruth, G.K.B.L.J.-P., Gouging elimination through tool lifting in tool path generation for five-axis milling based on faceted models. Int J Adv Manuf Technol - DOI 10.1007/s00170-005-0338-9, (2007): p. 293-309

9. Lauwers, B.K., G. Kruth, J. P., Development of a Five-axis Milling Tool Path Generation Algorithm based on Faceted Models. CIRP Annals Manufacturing Technology, Vol. 1, (2003): p. 85-88

10. Zill, D.G. and M.R. Cullen, "Definition 7.4: Cross product of two vectors". Advanced engineering mathematics (3rd ed.), Jones \& Bartlett Learning. p. 324. ISBN 0-7637-4591-X., 2006.

11. Zich, C.A.G.a.R.E., Cross product in N Dimensions the double wedge product. Politecnico di Milano, Energy Department, via La Masa 34, 20156 Milan, MI, Italy, (August 26, 2014) 